\title{
Rheumatoid Arthritis: The Problems Beyond the Joints
}

\author{
Hariprasad R \\ Junior Resident, Department of \\ Physiology, JIPMER, Puducherry, 605006, INDIA. \\ *Correspondence \\ Hariprasad R \\ Junior Resident, Department of \\ Physiology, JIPMER, Puducherry, 605006, INDIA. \\ Phone: +91-9952200808 \\ Email: hariprasadpims23@gmail.com \\ DOI: 10.5530/ijcep.2021.8.2.24
}

\section{NEWS}

\section{Rheumatoid Arthritis: The Problems beyond the Joints}

An autoimmune disease is defined as a clinical syndrome resulted from instigation of both $\mathrm{T}$ cell and B cell or individually, in the absence of any present infection or any sort of distinguishable cause. Rheumatoid arthritis is chronic and systemic autoimmune response to multiple joints with unknown etiology, progressive disability, systemic complications, early death and high socioeconomic costs. Rheumatoid arthritis is characterised by hyperplastic synovium, production of cytokines, chemokines, autoantibodies like rheumatoid factor $\left(\mathrm{R}_{f}\right)$ and anticitrullinated protein antibody (ACPA), osteoclastogenisis, angiogenisis and systemic consequences like cardiovascular, pulmonary, psychological and skeletal disorders. Cytokines a diverse group of polypeptides, play critical role in the pathogenesis of Rheumatoid arthritis. Their involvement in autoimmune disease is a rapidly growing area of biological and clinical research. Pharmacotherapy should target the balance between the pro and anti-inflammatory cytokines that is believe to be the central dogma in the pathogenesis of rheumatoid arthritis. ${ }^{[1]}$ Elevated sympathetic and parasympathetic nervous system activities are expected to occur as a consequence of inflammatory stress. ${ }^{[2]}$ For this we need to explore new treatment modalities to find the answers for unresolved etiology of autoimmune disease and to provide a quality of life to the patients suffering from rheumatoid arthritis.

\section{REFERENCES}

1. Alam J, Jantan I, Bukhari SNA. Rheumatoid arthritis: recent advances on its etiology, role of cytokines and pharmacotherapy. Biomed Pharmacother. 2017;92:615-33. doi: 10.1016/j.biopha.2017.05.055, PMID 28582758

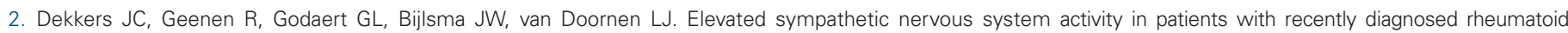
arthritis with active disease. Clin Exp Rheumatol. 2004;22(1):63-70. PMID 15005006.

\section{VIEWS}

\section{Yoga is Useful in the Management of Chronic Rheumatoid Arthritis}

Rheumatoid arthritis is not only a disease of joints and connectivetissue; instead it has its consequences in the limbic system, the sympathetic and parasympathetic nervous system.

In addition, autonomic nervous system (ANS) dysfunction may occur as a result of deconditioning mechanisms and psychological stressors such as the frequently occurring symptoms of rheumatoid arthritis - pain, fatigue and negative moods. These kinds of associations are likely to be bidirectional. For example pain as a stressor that induces sympathetic activation and sympathetic activation affects pain threshold and tolerance.

Mind-body intervention like yoga is considered to be a better alternative therapy for controlling chronic pain. Yoga seems effective in decreasing pain due to inflammation and increasing quality of life. Yoga aims to combine physical, psychological and behavioural components and to improve internal harmony by stretching and strengthening of muscles and ligaments, breathing techniques and meditation. Yoga is commonly considered to be an acceptable and safe intervention. Yoga has a strong effect on ANS and the type of effect is different for different yoga techniques. Yoga was found to be beneficial in improving lung functions in patients with bronchial asthma and nerve conduction in type 2 diabetes mellitus. There is a similar study in progress that describes the "Effect of 12 weeks yoga therapy on pulmonary function and nerve conduction in patients with rheumatoid arthritis - A randomised control trial. It is strongly believed that yoga will be quite useful in the chronic management of not only the problems of the joints, but also the CV risks attributed by psychophysical stress associated with it.

Cite this article: Hariprasad R. Rheumatoid Arthritis: The Problems beyond the Joints (News and views). Int J Clin Exp Physiol. 2021;8(2):106. 